BULLETIN Bulletin hispanique

HISPANIQUE Université Michel de Montaigne Bordeaux

112-1| 2010

Actes du Colloque « langue, littérature, littéralité »

\title{
Pour une autre lecture de El peregrino en su patria de Lope de Vega
}

\section{Philippe Meunier}

\section{OpenEdition}

\section{Journals}

Édition électronique

URL : http://journals.openedition.org/bulletinhispanique/1099

DOI : 10.4000/bulletinhispanique.1099

ISSN : 1775-3821

Éditeur

Presses universitaires de Bordeaux

Édition imprimée

Date de publication : 1 juin 2010

Pagination : 75-88

ISBN : 978-2-86781-692-5

ISSN : 0007-4640

Référence électronique

Philippe Meunier, «Pour une autre lecture de El peregrino en su patria de Lope de Vega », Bulletin hispanique [En ligne], 112-1 | 2010, mis en ligne le 01 juin 2013, consulté le 01 mai 2019. URL : http:// journals.openedition.org/bulletinhispanique/1099; DOI : 10.4000/bulletinhispanique.1099 


\title{
Pour une autre lecture de El peregrino en su patria de Lope de Vega
}

\author{
Philippe Meunier \\ Université Jean Monnet de Saint-Etienne
}

Cette lecture du Peregrino en su patria se propose d'aller au-delà de l'étiquette de roman d'aventures qui lui est couramment apposée. En partant du double constat du titre paradoxal et de l'absence d'auto sacramental à la fin du cinquième livre, il s'agira de montrer à la lumière de trois épisodes particuliers - le pèlerinage à Montserrat, le séjour dans l'hôpital des fous de Valence et la nuit fantasmatique dans un autre hôpital, refuge de pèlerins - comment le destin romanesque de Pánfilo épouse une autre trajectoire - allégorique - sous l'espèce d'une "révélation personnelle».

Esta lectura del Peregrino en su patria se propone ir más allá de la etiqueta de novela de aventuras que se le suele dar a la obra. Teniendo en cuenta la paradoja del título y la ausencia de auto sacramental al final del libro quinto, se trata de mostrar a la luz de tres episodios particulares - la peregrinación a Montserrat, la estancia en el hospital de los locos de Valencia y la noche fantasmática en otro hospital, refugio de peregrinos - cómo el destino novelesco de Pánfilo adopta otra trayectoria - alegórica - que se asemeja a una "revelación personal ".

This reading of the 'Peregrino en su patria' sets out to going beyond the label of the adventure novel, as usually applied to it. After pointing out the paradoxical title as well as the absence of auto sacramental at the end of the fifth book, I will focus on three particular episodes - the pilgrimage to Montserrat, the stay in Valencia's madhouse, the fantastical night in another hospital, a refuge for pilgrims - to show how Panfilo's fictional destiny follows an allegorical itinerary in the guise of "personal revelation".

Mots-clés : Roman d'aventures - Pèlerinage - Auto sacramental-Écriture allégorique - Onomastique.

$B H i$, Tome 112, n 1 - juin 2010 - p. 75 à 88. 
$\mathrm{D}$ atée de 1604, cette œuvre de Lope que j’ai choisie pour illustrer ce colloque sur la littéralité ne semble guère susciter l'engouement de la critique et encore moins celle du public, puisque l'unique édition de référence reste celle de Juan Bautista Avalle-Arce de $1973^{1}$ - rééditée telle quelle il y a trois ans - et que, sauf erreur de ma part, la seule traduction française, celle de Vital d'Audiguier remonte à 1614. Tout se passe comme si la défaveur dont est victime El peregrino en su patria était inversement proportionnelle au succès de la réponse cervantine à Lope : le roman posthume du Persiles (Los trabajos de Persiles y Sigismunda. Historia septentrional ${ }^{2}$ ) qui ne cesse d'être réédité depuis quelques années. Je ne crois pas que le $400^{\mathrm{e}}$ anniversaire de la publication du Peregrino ait modifié substantiellement le panorama critique, hormis le Lope en 1604, recueil de douze études ${ }^{3}$ qui ne sont d'ailleurs pas toutes consacrées à l'œuvre qui nous intéresse.

La relative désaffection dont souffre cette œuvre lopesque tient principalement à deux facteurs. Le lecteur a souvent du mal à s'expliquer la raison de cette avalanche d'érudition ${ }^{4}$ qui lui semble freiner très souvent le rythme de la narration romanesque, et il s'interroge sur le caractère non fortuit de ces digressions qui, par exemple, retardent toujours plus le dénouement. Le second facteur tient sans doute à une défense appuyée et continue d'une Espagne tridentine, qui serait responsable, selon Emilia I. Deffis de Calvo, de l'absence d'évolution intérieure des personnages :

Pánfilo y Nise ya se habian convertido al iniciarse la historia, y carecen de la capacidad de modificar su entorno, no critican ni proponen una realidad sustituta. Se encuentran a merced de un narrador que confirma $y$ difunde un complejo ideológico ya consagrado, que se quiere perdurable e inconmovible: la monarquía absoluta y el magisterio infalible de la autoridad papal ${ }^{5}$.

1. Lope de Vega, El peregrino en su patria, Madrid, éd. de Juan Bautista Avalle-Arce, Clásicos Castalia, n 55, [1973], 2006.

2. Je remarque en effet que contrairement au roman cervantin traduit par Maurice Molho aux éditions Corti, et plus récemment par Jean-Marc Pelorson dans le second volume des œuvres romanesques complètes de Cervantès dans la collection «Bibliothèque de la Pléiade » chez Gallimard, El peregrino en su patria n'a pas fait l'objet d'une traduction réactualisée.

3. Lope en 1604, Luis Alberto Blecua Perdices y Guillermo Serés (dir.), Barcelona, Editorial Milenio, 2004.

4. Voir Juan Bautista Avalle-Arce : "Bien cierto es que en El peregrino en su patria hay demasiada erudición, y también damasiadas aventuras, y demasiada comedia, lo que aconsonanta la novela a la vida-obra de su autor, que fue una afirmación de demasía ", "Sentido y género de El peregrino en su patria ", ibid., p. 78.

5. Viajeros, peregrinos y enamorados. La novela amorosa de peregrinación del siglo XVII, Ediciones Universidad de Navarra, Anejos de Rilce n² 28, Pamplona, 1999, p. 64. 
Cependant si cette constance des personnages ne fait guère de doute, la raison est peut-être à rechercher dans un projet d'écriture avant de l'assujettir à une militance idéologique quelle qu'elle soit.

Comme il est de bon aloi à l'époque, cette ouvre cultive et revendique une poétique du mélange des genres : à la fois roman d'aventures, avatar hispanique du roman grec dans la lignée des Éthiopiques (Aventures de Théagène et Chariclée) d'Héliodore, florilège de poèmes amoureux, profanes et religieux, et dont quatre des cinq livres qui la structurent sont étayés par 4 autos sacramentales, successivement "Viaje del alma ", "Bodas entre el alma y el amor divino ", "La Maya », enfin celui de " El hijo pródigo ». Une question s'impose donc, à laquelle le présent article tâchera d'apporter une réponse : pourquoi le cinquième livre est-il dépourvu d'une "representación moral » allégorique?

El peregrino en su patria relate les péripéties, plus exactement les « travaux", les épreuves que subit le couple des protagonistes, Pánfilo, originaire de la capitale de l'empire espagnol, et Nise, tolédane. Leurs pérégrinations et pèlerinages se déroulent explicitement durant la période de l'entre-deux siècles, 1599 et 1600, respectivement année des noces de Philippe III et de Marguerite d'Autriche, et année sainte, non pas vues dans une stricte continuité chronologique, mais sans que l'on puisse parler non plus, comme le fait Avalle-Arce, de " desliz cronológico [que] se produce por la necesidad narrativa de ensamblar bodas reales y año santo ${ }^{6}$. Peut-être conviendrait-il de parler de deux dates superposées d'un même artefact textuel susceptible de fonctionner en permanence à deux niveaux.

Soit le passage suivant, extrait du livre IV : il s'agit de la rencontre de deux pèlerines espagnoles dans la ville de Marseille, Nise, laquelle avec ses cheveux courts se fait aisément passer pour un jeune homme, et Finea qui n'est autre que la sœur du héros de l'histoire, Pánfilo :

"¿De qué tierra eres, hermosa española? " La peregrina le respondió: "Noble mancebo, yo soy del reino de Toledo". " ¿En qué lugar naciste?", dijo Nise. "En el mejor, fuera de su imperial cabeza, respondió ella, pues ha merecido por tantos años aposentar a sus reyes". "¿De Madrid eres?, replicó Nise: mira qué dos peregrinos nos hemos juntado, que yo soy de Toledo. "La peregrina entonces con un suspiro dijo: "Ahi nació la causa de mi peregrinación y desventura". "Bien echo de ver, respondió Nise, de tu hermosura y pocos años, que amor te debe de traer en estos pasos,

6. ed. cit., note 259, p. 193. 
y siendo hombre noble de quien te quejas, pongo en duda que yo no le conozca". "Sí harás, dijo la peregrina de Madrid, y créeme que así como te vi se me heló la sangre, porque eres el mismo rostro de mi enemigo ${ }^{7}$.

La chose est entendue depuis longtemps : il ne saurait donc y avoir de pèlerin ou de pèlerine qui ne subisse les affres de l'amour, au point que l'avant-dernière phrase du livre célèbre les deux pèlerins d'amour : " $i D i c h o s o s$ peregrinos de amor, que ya en su patria descansan, cumplido el voto! ${ }^{8}$; tout cela en vertu de la compétence lexicale du signifiant espagnol, "peregrinación ", qui se décline aussi bien comme pérégrination au gré des caprices de la fortune que comme pèlerinage selon un itinéraire précis, subordonné à une destination prévue d'avance ${ }^{9}$. Ajoutons que le personnage lopesque du pèlerin est lié à la perfection en ce sens que le roman pose l'existence de deux couples : Pánfilo et Nise, d'une part, Celio et Finea, d'autre part. Vus de façon chiasmatique, ces deux couples d'amoureux construisent à leur tour deux paires gémellaires, permettant tous les miroitements pour pallier l'absence de l'être aimé ou la séparation d'avec lui.

Ce qui pose problème - et un problème impossible à éluder car il conditionne l'entier du livre - est que le port de la bure et le départ en pèlerinage semblent être seulement un prétexte à la fuite des deux amants et à la légitimité de leur amour. Or, dans le cas du roman de Lope de Vega, on apprend au livre III grâce à un récit enchâssé rétrospectif - selon la poétique du roman grec-que le topos de la fuite pour contourner l'obstacle est dénoncé comme totalement artificiel, en l'occurrence comme ironiquement superflu, puisque l'époux que la famille a choisi pour Nise n'est autre que Pánfilo ${ }^{10}$. Certes, les deux héros ne le savent pas à ce moment-là de leur histoire, mais Pánfilo et, du coup, le lecteur l'apprennent relativement tôt dans l'économie

7. Ibid., p. 352.

8. Ibid., p. 481.

9. C'est ce que confime le titre glosé de la traduction de Vital d'Audiguier en 1614 : Les diverses fortunes de Panfile et de Nise. Où sont contenues plusieurs amoureuses et véritables histoires tirées du pèlerin en son pays de Lope de Vega.

10. Le " charitable jeune homme " qui recueille Pánfilo à la porte de l'hôpital des fous de Valence et qui est aussi pèlerin et étranger dans sa propre patrie, n'est autre que Celio, le frère de Nise. Sans que ce dernier le sache, Pánfilo se retrouve donc au spectacle de sa propre histoire : «Pero mira qué estraño engaño les sucedió a entrambos para principio de sus desdichas, que habiéndose dicho en Madrid que Pánfilo volvía de Flandes vino a noticia de mi padre y deseoso de casarle con Nise [...] la llamó un día y la dijo que tenía concertado de casarla, y [...] escribió a Madrid rogando a su madre de Pánfilo se le enviase a Toledo ", op. cit., p. 258. 
du livre, dans une invitation à reconsidérer les lois du roman d'aventures à la lumière des différents sanctuaires visités.

On l'aura compris, le paradoxe de la formule titulaire selon laquelle le Castillan Pánfilo se déplace tel un étranger dans son propre pays - selon l'étymon " peregrinus »- et est désigné par antonomase comme tel, Peregrino, avec majuscule à l'initiale, se résout en un programme d'écriture qui veut, par exemple, que l'habit en serge censé dissimuler et dérober le pèlerin aux dangers imminents, le rende encore plus suspect, et le transforme en la cible choisie, non pas des coups d'une fortune aveugle, mais d'une Providence divine sur laquelle il faudra nous interroger. Toujours est-il que dès le début Pánfilo, confondu avec d'autres " bárbaros ", d'autres étrangers sous l'espèce de bandits catalans, se retrouve en prison à la fin du premier livre :

No se hizo la prisión del Peregrino tan a poca costa de su paciencia, que [...] alcanzase su inocencia la libertad que merecía, ni su opinión buena fama, porque debajo de estar en aquel hábito daba sospecha a los jueces de que no carecía de culpa ${ }^{11}$.

Une fois libéré - parce que le capitaine des bandits, Doricleo, est réintégré dans la communauté des citoyens de Barcelone -, Pánfilo assiste aux fêtes de la ville, et en particulier, à une représentation d'un auto sacramental intitulé "Viaje del alma ", lequel met en scène la bataille navale entre l'embarcation de Plaisir dont la hune est occupée par Superbe, et celle de Pénitence dont le pilote est le Christ en personne. Il s'agit là, bien sûr, de la dramatisation du choix que doit faire l'âme qui finit par confier son libre-arbitre à Dieu. On remarquera que cette pièce eucharistique qui clôt le premier livre répond aux premières pages s'ouvrant sur le lieu commun du naufrage dont est victime sur la grève barcelonaise le protagoniste, alors séparé de son aimée. Non seulement la métaphore traditionnelle ${ }^{12}$ qui fait de la vie ici-bas une navigation périlleuse se trouve légitimée par le sens littéral, mais l'expression, c'est-à-dire le jeu théâtral et les péripéties propres au roman grec sont significativement rapprochées pour construire un contenu ${ }^{13}$ général et englobant qui reste

11. Ibid., p. 107.

12. Cette métaphore est aussi à l'œuvre dans la chanson qui ouvre la représentation théâtrale lors des fêtes consacrées à saint Jacques le Majeur dans la ville, espagnole alors, de Perpignan : "Y cuando el cuerpo llora el alma tiembla, / saca el sol de piedad las rubias trenzas, / y en una tabla de arrepentimiento / llega el cuerpo a la orilla, el alma al puerto. " Remarquons au passage que cette chanson introduit la pièce eucharistique consacrée à une parabole qui joue sur une autre modalité de pérégrination, celle de l'enfant Prodigue, ibid., p. 368.

13. Voir Paul Zumthor, Langue, texte, énigme, Paris, édition du Seuil, 1975, "Charles d'Orléans et le langage de l'allégorie», p. 197-213. 
encore à définir. Un autre exemple, d'ordre lexical celui-ci, permettra sans doute de mieux saisir la modalité de ce rapprochement : après avoir assisté à la représentation des noces de l'Âme et du Christ donnée à l'occasion du mariage royal, Pánfilo retrouve Nise à l'hôpital des fous de cette même ville de Valence et décide de s'y faire enfermer. Là, confiné dans une cage que le texte lopesque désigne sous le signifiant " gavia ", Pánfilo donne le spectacle de sa supposée folie devant un public composé de gentilshommes castillans et génois en partance sur les galères de Juan Andrea Doria, celles-là mêmes qui, évoquées dans la représentation de la pièce eucharistique, ont amené la reine Marguerite. C'est à ce moment-là que Pánfilo entreprend de décliner les différentes aptitudes référentielles du mot " gavia ":

Pues vea la gente sabia
si teniendo el árbol gavia
y siendo cárcel de seso
la gavia, se entiende en eso
cuánto es loco el que se fía
de la plaza o la crujia
de la nave o la galera ${ }^{14}$,

décrivant donc de façon explicite ce que fait le texte dans ce rapprochement systématique de l'auto sacramental et de la matière grecque de la prose, remotivant au passage l'étymon latin « cavea ", apte à référer à la partie du théâtre réservée aux spectateurs, et par synecdoque au théâtre tout entier.

On aura remarqué par ailleurs que Lope se plaît à " nationaliser » l'héritage d'Héliodore en privilégiant la côte méditerranéenne espagnole, la partie occidentale du bassin méditerranéen, en deux allers-retours du protagoniste entre les villes de Barcelone et de Valence, comme si l'espace géographique restreint était inversement proportionnel à l'intensité des aventures vécues :

Alli tomó Pánfilo su antiguo hábito, y [...] tomó segunda vez el camino de Barcelona, donde apenas hubo llegado cuando, siendo conocido de uno de aquellos hombres que habia herido en Monserrate, segunda vez fue preso y llevado donde los peregrinos alemanes lo habian estado hasta entonces. Caso digno de ponderación en cualquiera entendimiento discreto que un hombre no pudiese ni acertase a salir de tantas desdichas desde Barcelona a Valencia y desde Valencia a Barcelona, peregrinando en una pequeña

14. Op. cit., livre III, p. 281. 
parte de su patria España con más diversidad de sucesos que Eneas hasta Italia y Ulises hasta Grecia, con más fortunas de mar, persecuciones de Juno, engaños de Circe y peligros de lotófagos y Polifemos ${ }^{15}$.

Là encore, la raison est linguistique, sémantique, et tient à la compétence lexicale du signifiant " peregrino " : de par leur situation d'étranger et de pèlerin, les protagonistes deviennent des êtres insolites, hors du commun, proprement extra-ordinaires, qu'il s'agisse de la passion qu'ils vivent et qu'ils subissent, ou de leur beauté.

Comme l'indique la précédente citation, le premier sanctuaire où Pánfilo fait ses dévotions, est bien le temple catalan de Notre-Dame de Montserrat ; preuve que les aventures de notre protagoniste sont à considérer comme autant de pèlerinages, ou qu'elles soient associées dans un premier temps à ce type de voyage votif placé sous l'invocation mariale. Là, il rencontre deux autres pèlerins étrangers, l'un flamand, l'autre allemand, lesquels ont fui l'hérésie de la Réforme. Les trois personnages visitent de conserve le sanctuaire et, plutôt qu'une description exhaustive du spectacle des tapisseries et des ex-voto : linceuls, épaves de navires, chaînes et autres fers, l'épisode du pèlerinage à Montserrat est d'abord constitué par l'écriture de trois sonnets en latin, présentés comme traduits en l'honneur de la Vierge. Celui du pèlerin espagnol est retranscrit, en raison de sa valeur, en dernier ; en voici les deux tercets :

\section{¿Cómo tenéis, si os llama electro y rosa el Espejo, a quien dais tiernos abrazos, color morena, aunque de gracia llena? \\ Pero, aunque sois morena, sois hermosa, y ¿qué mucho si a Dios tenéis en brazos, que dándoos tanto sol, estéis morena? ${ }^{16}$}

Effectivement, cette sculpture romane polychrome de la fin du XII ${ }^{\mathrm{e}}$ siècle ${ }^{17}$ qui tient son fils dans ses bras, est appelée affectueusement par les Catalans la Moreneta, la Brunette, et peut être rangée parmi les vierges noires. En deçà de cette dénomination, il semble évident que le sonnet de Lope de Vega fonctionne bel et bien comme la glose, appuyée et redondante, du premier poème du Cantique des Cantiques récité par la Bien-Aimée :

15. Ibid., p. 365-366.

16. Ibid., p. 160-161.

17. Je rappelle qu'une chapelle consacrée à la Vierge exista dès 888 , à une époque où la Catalogne est sous domination maure. 


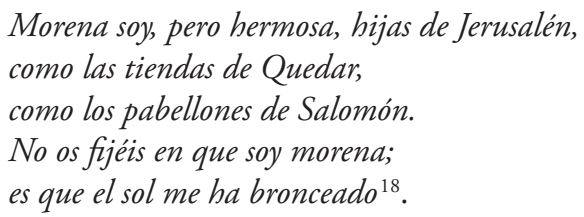

Je ne sais pas si une tradition poétique orientale à travers les textes bibliques aurait eu une influence sur la représentation de la Vierge Marie, mais ce qui est cohérent dans le texte lopesque, c'est que d'une part, à peu de frais, l'auteur distille comme un parfum d'un orient exotique sans que son personnage ait à sortir des frontières de la Péninsule Ibérique ${ }^{19}$, et que d'autre part, le sonnet de Pánfilo pose le problème du littéral et du figuré dans la continuité de l'exégèse biblique.

La seconde partie du pèlerinage est constituée par l'ascension de cette montagne escarpée, au rythme des neuf ermitages qui jalonnent le chemin et dans lesquels nos trois pèlerins écoutent des histoires exemplaires. Or, contre toute attente, le pèlerinage jusqu'au sommet de la montagne est interrompu : entre la huitième et la neuvième chapelle, le Flamand, l'Allemand et l'Espagnol redescendent à l'auberge du monastère pour y passer la nuit où l'un des deux étrangers du Nord est accusé de complicité avec une servante qui a volé des bijoux. Pánfilo défend ses frères d'habit et de religion en maniant le bourdon comme une épée et réussit à s'enfuir, comme si l'héritage d'Héliodore avait pris le pas sur l'ascension exemplaire, faite de renoncement, laquelle ne parviendra jamais à la plus haute chapelle, celle de Saint-Jérôme. Ce pèlerinage raconté dans le second livre, qui rate donc sa destination finale, va trouver en réalité sa complétude bien plus tard, dans le cinquième et dernier livre, lorsque le pèlerin espagnol se retrouve à la frontière des royaumes d'Aragon et de Castille. En fuite, car recherché pour un crime dont il est évidemment innocent, Pánfilo arrive dans une ville anonyme où personne ne veut l'héberger. La saleté de sa mise, sa chevelure hirsute, ses pieds en sang ont raison de l'hospitalité des autochtones, et le Peregrino n'a d'autre recours que de trouver refuge dans un hôpital étrangement vide, sans lumière, où il se dirige à tâtons à l'aide d'une houlette, à défaut de son bourdon qu'il a abandonné dans une péripétie antérieure. Cet espace fantomatique est pourtant habité par un anachorète qui vit recueilli dans une chapelle :

18. La Santa Biblia, traducción bajo la dirección de Evaristo Martín Nieto, Madrid, San Pablo, 1998, p. 918.

19. Pour ce qui est, bien sûr, du récit premier, et non des récits seconds enchâssés comme celui de Nise où elle raconte à Finea leurs aventures en Berbérie. 
[...] y vio Pánfilo un hombre de mediana estatura y edad, los cabellos largos y la barba crecida y enhetrada; cubriale una ropa de sayal hasta los pies. La capilla era pequeña, el retablo devoto y en la peana dél dormía aquel hombre; tenia por cabecera una piedra, su báculo por compañia y una calavera por espejo, que ninguno muestra mejor los defetos de nuestra vida ${ }^{20}$.

Le pèlerin espagnol a effectivement trouvé, après 300 pages pour le lecteur, l'ermitage de Saint-Jérôme, mais au prix de plusieurs inversions significatives. L'ascension escarpée comme chemin de douleurs n'a lieu qu'après, sous l'espèce d'une nuit terrifiante et cauchemardesque, expression fantasmatique non seulement de l'angoisse du personnage ${ }^{21}$, mais aussi d'une radicale et littérale dépossession de lui-même. Seul dans une grande pièce et malgré ses appels au secours à la Vierge de Guadalupe, le pèlerin castillan, l'Étranger dans sa propre patrie, voit comment deux hommes s'approchent de lui, l'un tenant un grand plat, l'autre aiguisant un couteau, sorte de prélude à sa propre décollation. Au moment où il avance les mains pour repousser l'arme fatale, il sent que ses deux membres antérieurs sont emportés, puis terrifié, il voit à la lumière d'une torche deux grands chiens tenant chacun dans leur gueule une de ses mains. Si les deux molosses disparaissent en l'entendant appeler le Christ à l'aide, Pánfilo a l'impression un peu plus tard que son lit s'élève jusqu'au plafond pour s'écraser ensuite par terre, qu'une main le retient pour éviter sa chute ${ }^{22}$ et que l'on tire sur ses deux jambes, au point d'avoir l'impression de n'être plus qu'un corps tronqué : "Cesó la luz de aquel fuego, y tirándole de las piernas también le pareció que le faltaban y que había quedado el cuerpo tronco y sin ellas ${ }^{23}$; une sorte de corps devenu littéralement tronc, morceau de bois qui réduirait l'Étranger dans l'épreuve de crucifixion, à n'être que le support de lui-même. On le voit, cette histoire à dormir debout, et parce qu'elle est cela, n'en est pas

20. Ibid., p. 441-442.

21. L'épisode est à relier à toutes les menaces de mort que doit affronter l'Étranger, en particulier le châtiment de décapitation à Barcelone lorsqu'il est accusé d'avoir tué un officier de justice, ou lorsqu'il est pris pour l'assassin d'un certain Godofre et qu'il encourt la vengeance de Tirso, le frère du défunt.

22. Il est difficile de ne pas relier la narration, à ce moment-ci, à l'histoire miraculeuse bien connue - Juan Bautista Avalle-Arce rappelle qu'elle a sa source dans le livre VII du Speculum historiale de Vincent de Beauvais -, que le pèlerin allemand raconte lors de l'épisode de Montserrat, à savoir l'intercession salvatrice de la Vierge qui retient par la main un peintre en train d'esquisser son portrait, suite à l'effondrement de son échafaudage, ibid., p. 153.

23. Le signifiant espagnol est " tronco " qui peut être lu d'abord comme le participe passé irrégulier de "troncar ", 'tronquer', 'couper', comme le rappelle la note de Juan Bautista AvalleArce, mais aussi comme un substantif, 'tronc', dans une sorte de parataxe, ibid., p. 445. 
moins drôle et, placée sous le signe de l'illusion des sens, tourne très vite à la farce. J'en veux pour preuve ce spectacle pyrotechnique qui semble comme multiplier les feux de la rampe ; les fenêtres qui s'ouvrent comme autant de loges d'où un public d'hommes et de femmes rit de bon cœur et lui lance de l'eau ${ }^{24}$; sans parler des planches du sommier, lesquelles assimilées à une scène mouvante, convertissent Pánfilo et l'ingénuité attachée à son nom, en l'objet privilégié de cette dramaturgie carnavalesque nocturne.

Cet épisode, trop rapidement évoqué ici, se trouve placé entre deux autres pèlerinages proprement dits, lorsque le protagoniste, je l'ai sous-entendu auparavant, revient enfin chez lui, en Castille, après toutes les aventures catalanes. Ce chemin de retour vient à passer par la ville aragonaise de Saragosse et son temple dédié à la Vierge du Pilier. Je rappelle que si ce sanctuaire apparaît dans l'ordre de la narration lopesque comme la seconde destination de pèlerinage, il constitue un centre unique car originel de la dévotion mariale. Selon une tradition qui remonte à des documents $\mathrm{du}$ XIII ${ }^{\text {e }}$ siècle, la Vierge serait apparue de son vivant le 2 janvier de l'an 40 de l'ère chrétienne, à l'apôtre saint Jacques qui prêchait l'Évangile sur les rives de l'Èbre. Le sillon de l'histoire et de la narration romanesques remonte donc en sens inverse le temps chrétien de la piété mariale. Et si l'évocation de Nuestra Señora del Pilar n'a lieu qu'à la fin du troisième livre, ce relatif retard est compensé par une triple récurrence, puisque ce ne sont pas moins de trois pèlerins qui visitent successivement le sanctuaire. Le premier à le faire n'est pas Pánfilo, mais son double, Celio, qui sur le chemin de la France pour rechercher Finea, arrive à Saragosse le jour où une représentation théâtrale est donnée sur le parvis de la cathédrale. Comme dans les précédents livres, une chanson sert d'aperture au spectacle de la pièce eucharistique ; il s'agit ici d'un romance qui décline tous les noms du Christ sous forme de généalogie biblique et répète en un refrain les deux vers suivants :

\section{Vos sois aquel Cupido de amor vendado y por amor vendido ${ }^{25}$.}

L'intérêt de ce distique repose sur une paronomase peu originale, il est vrai, ressassée à l'envi par toute la littérature auriséculaire espagnole, "vendado » / "vendido ", mais qui prend tout de même ici un relief particulier. En faisant du Christ un nouveau "Cupidon / d'amour aux yeux bandés et par amour vendu», Lope rapproche jusqu'à les confondre, explicitement cette

24. Ibid., p. 444.

25. Ibid., p. 283. 
fois-ci, l'amour divin et l'amour mondain des pèlerins, en accord avec le symbolisme de ce pilier ${ }^{26}$, ou plus précisément de cette colonne en jaspe, recouverte de bronze et d'argent, pour signifier la médiation de ce conduit entre le ciel et la terre.

Le second pèlerinage au sanctuaire aragonais est accompli par l'Étranger dans le dernier livre, je l'ai dit, alors qu'il est poursuivi pour un meurtre qu'il n'a pas commis. Seul et contre tous, le pèlerin élève un chant de louanges à la Vierge. Sous la forme de huitains hendécasyllabiques, il invoque celle dont l'humble enfant est venu réchauffer l'immensité de l'univers :

\section{Paloma celestial, en cuyo nido, envuelto en pobres paños cupo al yelo aquel sol que midió sin ser medido, la tierra, el mar, el aire, el fuego ${ }^{27}$.}

À ce moment-ci de l'histoire, il semble difficile de ne pas remarquer l'analogie entre le dénuement de la naissance du Christ et celui qui, de retour dans sa terre natale, vit ses aventures comme un dénudement spirituel. Et cette idée me semble confirmée par le troisième pèlerinage à la cathédrale de Saragosse à la toute fin du livre, accomplie cette fois-ci, par le double féminin de Pánfilo, c’est-à-dire Nise, celle qui est enfin dénommée, elle aussi par antonomase, "la Peregrina de su patria ${ }^{28}$. L'évocation très rapide est compensée par la valeur symbolique de retour aux origines du culte marial, de retour à un état premier. Si ce sanctuaire est sur la route qui doit ramener à la maison familiale de Tolède, c'est aussi l'endroit où se termine la pérégrination de l'héroïne qui se défait de son habit de pèlerinage et de son bourdon laissés en ex-voto sur les murs de la chapelle dédiée à la Vierge du Pilier.

Reste encore un troisième et dernier pèlerinage, je l'ai dit, celui effectué par Pánfilo au monastère de Guadalupe, réduit à un sonnet de louanges mariales, mais dont le principal intérêt est d'être surtout celui qui fait partie du royaume de Castille d'où sont originaires les protagonistes ; un sanctuaire qui géographiquement fait partie de l'histoire première et personnelle des

26. Selon la tradition, la Vierge serait apparue à saint Jacques sur un pilier en marbre qui serait resté pour marquer l'apparition miraculeuse et l'emplacement de l'église à construire. C'est ce que rappelle le narrateur au moment où Pánfilo fait ses dévotions à la Vierge du Pilier, p. 440.

27. Ibid., p. 440.

28. Ibid., p. 470. 
personnages ; celui qui est le plus près de l'ancienne capitale impériale où est célébrée la concorde finale ${ }^{29}$.

Une conclusion provisoire s'impose donc au terme de cette rapide description qui permet de comprendre pourquoi le dernier livre n'est pas couronné par un auto sacramental, et pourquoi dans le type de langage mis en place par Lope de Vega, l'on ne saurait dissocier les aventures en prose du couple des pèlerins de l'écriture métrée des pièces eucharistiques et des poèmes.

Les retrouvailles de Pánfilo et de Nise peuvent être entendues comme le dénouement allégorique des noces entre Dieu et l'Âme, venant subsumer ainsi les quatre autos sacramentales des livres précédents; et en ce sens, il ne saurait y avoir d'évolution des personnages ${ }^{30}$. Si l'on considère le langage de l'allégorie comme l'association d'une personnification de réalités abstraites et d'une métaphore prolongée, les péripéties "à la mode " du roman grec vécues par les deux héros constitueraient donc le plan littéral, l'expression, qui à son tour permet de remotiver le signifiant onomastique et de retrouver derrière l'étymologie savante ou ludique, le plan du figuré, le contenu. Pánfilo, dont je lis le nom-valise comme la conjonction de l'adverbe $\pi a \nu$, 'tout', 'entièrement' et du verbe $\Phi_{l} \lambda \varepsilon \imath \nu$, 'aimer' à la première personne du présent de l'indicatif, n'existe comme personnage que parce qu'il personnifie l'idée abstraite d'amour universel. Quant à Nise, son nom qui a déjà été l'objet d'un sonnet " equívoco ${ }^{31}$, peut être lu de manière anagrammatique comme Inés, la désignant par étymologie comme l'incarnation de la chasteté. Mais il y a mieux encore : le prologue de la troisième pièce religieuse, celle qui met en scène l'Âme comme épouse du Christ, dans sa version proprement

29. C'est d'ailleurs ce que souligne le narrateur lors de la nuit de cauchemar dans l'hôpital des pèlerins abandonné à la frontière de l'Aragon et de la Castille : « [...] el mísero Pánfilo comenzó a llamar a la virgen de Guadalupe, que sólo le faltaba de visitar en España, aunque era del reino de Toledo, porque las cosas que están muy cerca, pensando verse cada día, suelen dejar de verse muchas veces ", ibid., p. 443.

30. C'est à ce titre que les matières narrative et lyrique - mais encore faut-il le démontrer " indudablemente conforman un todo significativo junto con los autos sacramentales ", Emilia I. Deffis de Calvo, op. cit., p. 61.

31. Voir livre V, le sonnet de Leandro : « Ni sé si vivo, ni si estoy muriendo, / Ni sé qué aliento es éste, en que respiro, / Ni sé por dónde a un imposible aspiro, / Ni sé por qué razón amando ofendo. / Ni sé de qué me guardo o qué pretendo, / Ni sé qué gloria en un infierno miro, / Ni sé por qué sin esperar suspiro, / Ni sé por qué rendido me defiendo. / Ni sé quién me detiene o quién me mueve, / Ni sé quién me desprecia o me recibe, / Ni sé a quién debo amor o quién me debe, / mas sé que en estas cuatro letras vive / un alma sin piedad, un sol de nieve, / que yela y quema y en el agua escribe », op. cit., p. 468. 
espagnole du personnage de la $\mathrm{Maya}^{32}$, permet de privilégier la première syllabe du nom du héros. La chanson prologale joue en effet par homonymie et homographie sur le signifiant "Pan " avec majuscule à l'initiale pour désigner le dieu musicien dont Covarrubias commente ainsi l'emblème d'Alciat : "[...] entendiéndose por él toda la fábrica del cielo y mundo ", et celui dont le sacrifice est célébré à travers le pain eucharistique :

Las siete voces que Pan
juntó de cañas y cera,
fue la música postrera
que de vos oyendo están.
Y si allá quedó vencido
Pan de Apolo, vos Pan solo
con esta música a Apolo
dejasteis escurecido.
[...]
Yasí justamente a vos
de Dios Pan el nombre os dan,
Porque ser Dios y ser pan,
¿quién puede ser sino Dios?33

Dès lors s'éclaire le paradoxe titulaire de "l'Étranger dans sa patrie » : seul un personnage christique qui est plénitude amoureuse peut être étranger à lui-même au point de faire don de soi. C'est en ce sens que l'on comprend que la pérégrination de Pánfilo est une « régression », sacrifice filial et retour à la maison d'un père d'emprunt ${ }^{34}$.

Je terminerai par une objection que l'on est en droit de me faire : contrairement à l'auto sacramental et à ses personnages qui imposent la personnification et la métaphore filée, rien, si ce ne sont ces inversions que j'ai pointées du doigt, n'empêche le lecteur de rester sur le plan littéral des

32. $\mathrm{Ou}$ " Reina de Mayo ». Il s'agit comme le nom l'indique d'une fête printanière, urbaine et populaire où l'on élit la plus belle jeune fille - vierge - du quartier, que l'on pare d'une robe brodée d'or et d'argent et que l'on installe près d'un autel, telle une souveraine sur son trône. Les autres jeunes filles du quartier sont chargées d'attirer les passants pour qu'ils versent une obole. Voir José Deleito y Piñuela, También se divierte el pueblo, Madrid, Alianza Editorial, 1998, p. 25. Voir aussi le bel ouvrage de Julio Caro Baroja, La estación de amor, Madrid, Taurus, 1983, en particulier le chapitre 8 de la première section consacrées aux fêtes de mai, p. $52-53$.

33. Prologue à l'Auto de La Maya, livre III, op. cit., p. 285-286.

34. On se rappellera que Pánfilo retourne comme palefrenier chez le père de Nise chez qui il a déjà servi comme domestique. 
aventures du couple protagoniste et de refuser toute exégèse. C'est bien là toute l'originalité de Lope qui feint de renouer avec la poétique du roman d'aventures pour mieux circonvenir ou détourner l'héritage d'Héliodore, en faisant en sorte, comme dans les grands textes de la fin de l'époque médiévale, que « le littéral confère au figuratif un aspect si concret et une telle force de crédibilité que toute idée abstraite s'efface et que l'allégorie prend la valeur d'une révélation personnelle $»^{35}$. En dernière instance, la figure du pèlerinage est celle de la lecture et la capacité du lecteur à découvrir, derrière le chatoiement artificiel des rebondissements de l'action, la signification profonde de l'œuvre : capacité du lecteur à se laisser guider jusqu'au sens révélé. 